\title{
Final Technical Report for the Grant entitled "Genetic Factors Affecting Susceptibility to Low-Dose Radiation"
}

This is the terminal report for the project "Genetic Factors Affecting Susceptibility to Low-Dose Radiation" DOE project DE-FG02-99/ER6285902ER62859. The goal of the proposal was to test the hypothesis that mice heterozygous for the NBS1 gene were genetically susceptible to low doses of ionizing radiation.

Rationale: Risk estimates for radiation exposure are based on the whole population. There is evidence that there may be subpopulations within the human population that are genetically susceptible to ionizing radiation, e.g., patients with the disorders of Nijmegen Breakage Syndrome (NBS) and Ataxia telangectasia (AT). These patients are radiation sensitive because of defects in cellular responses to radiation induced genetic damage. It is unclear whether humans heterozygous for the mutations associated with NBS or AT are radiation sensitive and results from cell culture experiments give conflicting results. NBS and AT patients are easily identified but obligate heterozygotes for these disorders, estimated to be $1-4 \%$ of the US population, are subpopulations that make up a considerable fraction of the US population that may be susceptible to low doses of radiation.

Hypothesis to be tested: that animals with genetically defined haploinsufficiency at the NBS1 loci are predisposed to radiation sensitivity.

The model system: In collaboration with John Petrini at the Memorial Sloan Kettering Cancer Center in New York City we developed a mouse model of this disorder (Williams B.R., Mirzoeva, O.K., Morgan, W.F., Lin, J., Dunnick, W. and Petrini, J.H.J. A murine model of Nijmegen breakage syndrome. Current Biol. 12: 1-6, 2002). These animals have an induced genetic disruption that makes a 
deletion in NBS1 exon 5, that closely mimics the disorder found in the vast majority of human patients with NBS.

Experimental design: Mice were exposed to $0,1,10$ or 100 cGy of X-rays 8 weeks after birth and monitored for delayed radiation effects. This was a life time study, and we used analysis of micronuclei to determine whether hypomorphic animals showed increased evidence of radiation-induced genomic instability and tumor induction to determine potential sensitivity. Animals were routinely monitored until they died from natural causes, or were euthanized because of other detrimental health effects (see below). NOTE: death was not an endpoint in the proposed experiments. Any animals with palpable tumors, become moribund, or show signs of discomfort (lethargy, weight loss, or unkempt appearance) were sacrificed. Furthermore, mice are monitored twice weekly for weight loss, respiration, eye color, potential abdominal extension, cachexia, rough coal hair, dehydration, and/or tumor induction; and every 4-6 months for micronuclei.

Results: This study is now complete and all animals in the cohort are now deceased.

Analysis of micronuclei was used to investigate potential delayed genomic instability occurring in irradiated animals. At regular intervals post irradiation blood samples are taken from all animals using tail vein sticks, and the frequency of micronuclei per 2000 polychromatic erythrocytes determined. There were no significant differences in micronuclei frequencies between irradiated and nonirradiated animals, or between wild type and our hypomorphic NBS mice. We did however, notice a small, but not statistically significant increase in the frequency of micronuclei as the animals age.

There was also no increase in tumor incidence between wild type and heterozygous animals after irradiation. Animals were followed until they died 
from natural causes or in rare cases had to be euthanized upon advice of the animal care staff because of animal discomfort. All the animals are now deceased and the codes have been broken. There was no non-specific life shortening associated with low dose radiation exposure in either wild type or heterozygous animals when compared with the non irradiated control animals, and if anything the 10cGy irradiated NBS1+/- animals lived longer than both the non-irradiated control and NBS1+/- animals. These data are currently being analyzed by statistical staff at the University of Maryland to determine potential statistical significance, if any, of low dose radiation induced genomic instability, tumor induction, or life shortening. However it is clear from the data that even if there are differences between the wild type and NBS+/- mice these differences will be very small at best.

We also used these animals to determine if we could develop a rapid and sensitive assay for identifying individuals heterozygous for NBS in the human population. Our goal was to develop a functional assay by which heterozygotes can be distinguished from DNA polymorphisms in order to identify potentially sensitive individuals. We used both qualitative and quantitative Mre11/Rad50/NBS1 foci formation as a function of radiation dose and could never reliable identify NBS heterozygotes despite detailed genomic analysis. We also investigated the cytogenetic G2 assay as a method of identifying NBS+/m individuals but we did not find a consistent or statistically significant difference in chromatid aberrations between NBS $+/+$ and $\mathrm{NBS}+/ \mathrm{m}$. Animals with two mutant NBS alleles were identifiable using this technique, but this is obviously not relevant to the human situation.

We have also completed a series of low dose irradiation experiments in collaboration with Peter McKinnon at St. Jude's to investigate potential changes in gene expression. Mice were irradiated and the indicated organs (brain and thymus) were harvested for microarray analysis. The data obtained are currently being compared to the previously established data set from ATM deficient mice 
in the Petrini laboratory at Memorial Sloan Kettering Cancer Center in New York City. A second set of animals was sent to St. Judes, after we determined that the initial samples from brain and thymus were contaminated to some extent with irrelevant tissue. Nevertheless, the data obtained in the first set of experiments provided some information, as contaminating signal can in some cases, being easily discerned and discarded. Analysis of this second data set is now underway.

Most recent products delivered:

Williams B.R., Mirzoeva, O.K., Morgan, W.F., Lin, J., Dunnick, W. and Petrini, J.H.J. A murine model of Nijmegen breakage syndrome. Current Biol. 12: 1-6, 2002

Most recent notes concerning the project:

We anticipate two more manuscripts to result from this study. The first will be on the results of the low dose radiation study in these hypomorphic mice and we are awaiting the results of the statistical analysis. The second manuscript will be on the low dose radiation induced gene expression studies and we are awaiting analysis of the second, hopefully much cleaner data set from MSKCC. 